\title{
A transaction-based method for allocation of transmission grid cost and losses
}

\author{
Julio Usaola* \\ Departamento de Ingeniería Eléctrica of Universidad Carlos III de Madrid, Av. Universidad 30, Leganés, 28911 Madrid, Spain
}

\begin{abstract}
The problem of grid cost and losses allocation may be divided into independent subproblems: allocation of branch flow and losses to transactions, definition of these transactions and cost allocation to transactions. From this final allocation, the charges to participants in transactions may be made straightforwardly. A differential, slack-invariant method for the allocation of flow and losses to transactions that makes use of the AC load flow equation is presented here. The definition of transactions must be addressed using a non-discriminatory rule in pool systems. There are many possible options for this definition, and the choice made has great influence on the results. Cost allocation, on the other hand, may be made in different ways, as well. The paper presents an allocation process that addresses all these issues. Results for the IEEE-RTS96 test system are obtained and discussed.
\end{abstract}

Keywords: Transmission grid cost allocation; Loss allocation; Differential method

\section{Introduction}

Transmission cost and losses allocation are different though related problems in deregulated electricity markets. The chosen allocation procedures for both problems must provide locational signals and incentives in order to encourage efficient use of the transmission facilities. They must also be based on actual grid use. The proposed methods in technical literature for solving these problems share several points.

Transmission cost allocation methods must comply with some conditions, namely to avoid cross-subsidies, to be transparent and easy to implement, to ensure cost recovery, to provide adequate economic signals and to have continuity with time [1]. The proposed methods could be classified as embedded cost and marginal cost methods. The latter, however, do not guarantee cost recovering in real networks [2]. Embedded cost methods, on the other hand, allocate the transmission costs according to the extent of use of generators and consumers. Several methods of this kind have been proposed and are in use in different systems. They can be divided into rolled-in methods and load

\footnotetext{
* Tel.: +34 916249404; fax: +34 916249430 .

E-mail address: jusaola@ing.uc3m.es.
}

flow-based methods. Rolled-in methods charge a fixed amount per energy unit, and their main drawback is that they ignore actual network use and that they do not send adequate economic signals to grid users. Flow-based methods, on the other hand, charge the users in proportion to the use they make of grid facilities. Some proposed methods of this kind may be classified as proportional [3] or differential methods [4].

Loss allocation methods may also be classified as proportional [3], differential [5-8], circuit-based [9,10] and others [11]. It must be outlined that this problem has no unique solution, due to its non-linearity, and some assumptions have to be made for any possible allocation. Among the proposed methods, the proportional method has several advantages: it is simple to understand and provides several results such as loss allocation, grid use and load sharing among generators. However, although it begins from a solved load flow, it does not follow the Voltage Kirchhoff Law in the allocation process, ignores the counterflows, and the results seem to be too volatile [4]. Differential (or incremental) methods are, on the other hand, well known in literature and are based on the Incremental Loss Coefficients [12]. These coefficients, however, depend on the choice of the slack bus in the studied case, and, therefore, there is a part of arbitrariness in the allocation. Several proposals have been made to overcome this difficulty. In [7], a fictitious slack bus is cho- 
sen; in $[5,6]$, the property of invariance of the allocation for a transaction is applied, claiming a total invariance of the slack bus choice.

Another method for overcoming this difficulty, but applied to the transmission cost allocation method for bilateral exchanges, is given in [4]. This method allocates transmission costs among transactions, obtaining participation factors in the transmission network for each one of these transactions, and assuming that the generator node is a slack bus in each transaction. It is only applied to lossless systems and uses DC load flow equations.

In this paper, the problems of transmission grid cost and losses allocation to transactions are divided into three subproblems that can be addressed independently. First, the definition of grid users. Second, the allocation of power flows through branches and losses to each grid user, and finally, the cost allocation to the already allocated flow and losses. Different solutions can be given to all of them.

The grid users to whom grid use and losses are allocated is a very important question. In this paper, the allocation is made to transactions (generation-load couples), in order to make it invariant from the slack bus choice of the initial load flow. It must be recalled that when using differential methods for direct allocation to nodes (for instance, with Incremental Loss Coefficient), a transaction is tacitly assumed between each node and the slack node.

The definition of the transactions is straightforward for markets based on bilateral contracts, but in pool organized markets a definition of transaction must be made. This definition has a great influence on the results, as will be shown in this paper. Two principles for defining these transactions in a pool-based system that have been proposed in literature are examined in this paper. These methods are the Proportional Sharing Principle (PSP) and the Equivalent Bilateral Exchanges (EBE). The consequences of this choice have been analyzed.

The grid use and loss allocation problems are solved in this paper using a differential method for the allocation of branch flows and losses to transactions. The method is slack invariant that is to say that the results do not depend on the choice of the slack bus of the system in the initial load flow. AC load flow equations are used, allowing to solve both (power flow and losses) allocation problems with the same method. The use of $\mathrm{AC}$ load flow equations involves more theoretical complexity in the formulation, but does not imply a significative increase in computation time. Also, by using the AC load flow equations, a greater accuracy can be expected.

The cost allocation problem has also been addressed, and different solutions have been considered. A further decision must be taken about the percentage of costs allocated to generation and load. In non-slack-invariant methods, this choice is taken by choosing a slack node close to the generation or load centers. However, the fact that the costs are allocated to transactions, and from this to users, allows more flexibility.

The main contributions of this paper are intended to be the following:

- The split of the whole problem into three subproblems: transaction definition, use of grid and losses allocation, and cost allocation, with different possible solutions for each of them. This division allows to make choices for each subproblem being aware of their consequences.

- The proposal of a method for flow allocation to transactions that is differential and slack invariant (DSI method). This method makes use of the AC load flow equations.

- A method (parallel to the previous one) for loss allocation that is also slack invariant.

- A study about the consequences of the definition of transactions in pool systems, together with a proposal of the choices that could be considered more reasonable.

The paper is organized as follows. Section 2 exposes the differential, slack-invariant (DSI) method of flow and losses allocation to transactions. Section 3 deals with the problem of cost allocation to transactions, while in Section 4 the subject of transaction definition choice is addressed. In Section 5 , the numerical results of the application of the method to the IEEE 24 nodes Reliability Test System [16] are given and commented.

\section{Flows and losses allocation}

\subsection{General formulation}

The method begins from the results of a solved AC load flow in a system with a given load and generation. This solution is used as a start point.

The average power flow and losses in branch $r$ are given by Eqs. (1) and (2). All the symbols are explained in Appendix B.

$$
\begin{aligned}
& F_{r}=\frac{1}{2} g_{k m}\left(u_{k}^{2}-u_{m}^{2}\right)-b_{k m} u_{k} u_{m} \sin \left(\delta_{k}-\delta_{m}\right) \\
& L_{r}=g_{k m}\left[u_{k}^{2}+u_{m}^{2}-2 u_{k} u_{m} \cos \left(\delta_{k}-\delta_{m}\right)\right]
\end{aligned}
$$

Let us define $T_{i j}$ as the transaction $t$ of a power $P_{i j}$ between the generation node $j$ and the demand node $i$. Differential methods allocate these losses and power flow to transactions from the value of sensitivities of average flow $\left(\phi_{r t}\right)$ and losses $\left(\lambda_{r t}\right)$ to a differential increase in each transaction $t, T_{i j}$. These sensitivities will be obtained in Section 2.2. Hence, the increment of average flow of branch $r, \mathrm{~d} F_{r t}$, and the increment in losses, $\mathrm{d} L_{r t}$, due to the differential transaction $t$, could be found as (3).

$$
\begin{aligned}
& \mathrm{d} F_{r t}=\phi_{r t} \mathrm{~d} T_{i j} \\
& \mathrm{~d} L_{r t}=\lambda_{r t} \mathrm{~d} T_{i j}
\end{aligned}
$$

These equations are only valid for differential variations in transactions. In order to obtain the allocation of the flow to a transaction, it would be necessary to integrate them somehow. The integration process requires an initial point, to define an integration path $I_{\mathrm{P}}$, and to know the value of the sensitivities along this path, $\phi_{r t}\left(T_{i j}\right), \lambda_{r t}\left(T_{i j}\right)$. A discussion about this point will be given in Section 2.3.

If all these aspects are defined in some way, the flow in branch $r$ due to transaction $t, F_{r t}$, along the integration path $I_{\mathrm{P}}$, could be 
found by (4).

$$
F_{r t}=\int_{I_{\mathrm{P}}} \phi_{r t}(t) \mathrm{d} t
$$

While the losses in line $r$ due to transaction $t$, along the integration path $I_{\mathrm{P}}, L_{r t}$, are given by Eq. (5)

$$
L_{r t}=\int_{I_{\mathrm{P}}} \lambda_{r t}(t) \mathrm{d} t
$$

Some points must be highlighted:

- The independence of the slack bus is given (under certain conditions explained in Section 2.2) by the fact that the sensitivities are found with respect to transactions.

- In Section 2.3, an alternative approach will be given-a reconciliation process will be proposed instead of the integration. The assumptions under which this reconciliation gives the same results as the integration process will be also shown in the same section.

- Once the allocation to a transaction is made, it may be necessary to share it between generation and load. This may be made in several ways, by agreement between agents, in the case of a bilateral contract, or by dividing it between them in some way. This problem could be also solved by using the Incremental Loss Coefficients to allocate losses directly to nodes, and choosing an appropriate slack bus for dividing this allocation into generation and demand. This solution is less flexible than the proposal made here, where the share could be even different for each transaction. Besides, in systems with no clear spatial separation between generation and load, is not straightforward to choose the adequate slack bus for this purpose.

In the next section, the derivation of the sensitivity terms is made.

\subsection{Sensitivity terms}

The terms $\phi_{r t}$ and $\lambda_{r t}$ can be obtained as (6) and (7).

$$
\begin{aligned}
\phi_{r t}= & {\left[g_{k m} u_{k}-b_{k m} u_{m} \sin \left(\delta_{k}-\delta_{m}\right)\right]\left(\frac{\partial u_{k}}{\partial P_{j}}-\frac{\partial u_{k}}{\partial P_{i}}\right) } \\
& -\left[g_{k m} u_{k}+b_{k m} u_{k} \sin \left(\delta_{k}-\delta_{m}\right)\right]\left(\frac{\partial u_{m}}{\partial P_{j}}-\frac{\partial u_{m}}{\partial P_{i}}\right) \\
& -b_{k m} u_{k} u_{m} \cos \left(\delta_{k}-\delta_{m}\right) \cdot\left[\left(\frac{\partial \delta_{k}}{\partial P_{j}}-\frac{\partial \delta_{k}}{\partial P_{i}}\right)\right. \\
& \left.-\left(\frac{\partial \delta_{m}}{\partial P_{j}}-\frac{\partial \delta_{m}}{\partial P_{i}}\right)\right]
\end{aligned}
$$

$$
\begin{aligned}
\lambda_{r t}= & {\left[2 g_{k m} u_{k}-2 g_{k m} u_{m} \cos \left(\delta_{k}-\delta_{m}\right)\right]\left(\frac{\partial u_{k}}{\partial P_{j}}-\frac{\partial u_{k}}{\partial P_{i}}\right) } \\
& +\left[2 g_{k m} u_{m}-2 g_{k m} u_{k} \cos \left(\delta_{k}-\delta_{m}\right)\right]\left(\frac{\partial u_{m}}{\partial P_{j}}-\frac{\partial u_{m}}{\partial P_{i}}\right)
\end{aligned}
$$

$$
\begin{aligned}
& -2 g_{k m} u_{k} u_{m} \sin \left(\delta_{k}-\delta_{m}\right) \cdot\left[\left(\frac{\partial \delta_{k}}{\partial P_{j}}-\frac{\partial \delta_{k}}{\partial P_{i}}\right)\right. \\
& \left.-\left(\frac{\partial \delta_{m}}{\partial P_{j}}-\frac{\partial \delta_{m}}{\partial P_{i}}\right)\right]
\end{aligned}
$$

where it must be noted that:

- The differential transaction $\mathrm{d} T_{i j}$ consists of an injection $\mathrm{d} P_{i j}$ in node $i$ and another, of the same value and opposite sign, in node $j$.

- The sensitivities of voltages modules $\left(u_{n}\right)$ and angles $\left(\delta_{n}\right)$ of node $n$ with respect to a power injection in nodes $i$ and $j$ are the terms of the inverse of the Jacobian matrix of the system.

However, the difference between two sensitivities depends on the slack bus choice in the initial load flow. Actually, the relation between the values of the difference of this value, when two slack buses o and s are considered, is given by Eq. (8)

$\left.\frac{\partial u_{k}}{\partial P_{j}}\right|_{\mathrm{o}}-\left.\frac{\partial u_{k}}{\partial P_{i}}\right|_{\mathrm{o}}=\left.\frac{\partial u_{k}}{\partial P_{j}}\right|_{\mathrm{s}}-\left.\frac{\partial u_{k}}{\partial P_{i}}\right|_{\mathrm{s}}-\left.\frac{\partial u_{k}}{\partial P_{\mathrm{o}}}\right|_{s} \frac{\mathrm{d} P_{1}}{\mathrm{~d} P_{i j}}$

where the subscript 'o' identifies the slack bus under which the load flow is run. The last term in the formula is the sensitivity of system losses $P_{1}$ to the transaction $T_{i j}$.

This relation, for the angle terms, is given by (9)

$$
\begin{gathered}
\left(\left.\frac{\partial \delta_{k}}{\partial P_{j}}\right|_{\mathrm{o}}-\left.\frac{\partial \delta_{k}}{\partial P_{i}}\right|_{\mathrm{o}}\right)-\left(\left.\frac{\partial \delta_{m}}{\partial P_{j}}\right|_{\mathrm{o}}-\left.\frac{\partial \delta_{m}}{\partial P_{i}}\right|_{\mathrm{o}}\right) \\
=\left(\left.\frac{\partial \delta_{k}}{\partial P_{j}}\right|_{\mathrm{s}}-\left.\frac{\partial \delta_{k}}{\partial P_{i}}\right|_{\mathrm{s}}\right)-\left(\left.\frac{\partial \delta_{m}}{\partial P_{j}}\right|_{\mathrm{s}}-\left.\frac{\partial \delta_{m}}{\partial P_{i}}\right|_{\mathrm{s}}\right) \\
-\left(\left.\frac{\partial \delta_{k}}{\partial P_{\mathrm{o}}}\right|_{\mathrm{s}}-\left.\frac{\partial \delta_{m}}{\partial P_{\mathrm{o}}}\right|_{\mathrm{s}}\right) \frac{\mathrm{d} P_{1}}{\mathrm{~d} P_{i j}}
\end{gathered}
$$

where the terms have similar meanings. These equations are demonstrated in Appendix A.

Eqs. (8) and (9) show that there is a difference between using one or another slack bus in the initial load flow. Three possible solutions could be given in order to make the proposed method slack invariant:

(1) To neglect this term, since it is a second-order differential term.

(2) To include the differential losses in the generating node $j$ of transaction $\mathrm{d} T_{i j}$. This would be similar to the method proposed in [4] for the lossless case. This possibility, however, must be coherent with the definition of transaction in lossy systems, since the losses produced by each transaction should be included in it. It must be recalled, however, that for lossless systems this term does not exist, and therefore, in these systems, the differences of sensitivities are slack invariant.

(3) Although the sensitivity terms could be calculated inverting the given Jacobian matrix, they can also be obtained numerically running incremental load flows, where the sensitivities, together with the terms $\mathrm{d} P_{1}$, could be found. A complete invariance could be achieved by running these incremental 
load flows with a distributed slack bus, where the differential losses would be assumed by all the nodes, except the generating node in the transaction.

If solution (2) or (3) is chosen for calculating the sensitivities, the method is fully slack invariant, as it will be shown in Appendix A. Actually, the differences in the results between adopting any of the three solutions is very small.

\subsection{Integration process. Reconciliation}

As explained in [6] about losses, the choice of different integration paths $I_{\mathrm{P}}$ would lead to different flow and losses allocations. One path could be to consider the variation of each transaction $T_{i j}$ from zero to its actual value while the rest of them remain at the same level. The sensitivity coefficients of Eqs. (6) and (7) would be different for each point along the path (i.e. a value of $T_{i j}$ ) and should be calculated, increasing the required computation time. Another possibility is to assume that all the transactions are zero and to raise them all at the same rate up to their actual values. Sensitivity coefficients should be also found for each point along the integration path, but the required computational effort is smaller in this case.

In any case, this integration process must be numerical, since no closed expression exists for the sensitivity coefficients as functions of the values of the transactions. Due to the almost quadratic dependence of losses with power, the trapezoidal rule of integration seems very appropriate for this purpose, because it reduces the number of integration steps for the same accuracy. It must be remarked that to follow this integration process, different fictitious situations must be run and analyzed. These situations can be unrealistic in many cases, especially when the transactions have a low value.

A good compromise is to follow a reconciliation process. This method is equivalent to an integration, using the implicit Euler integration rule, taking only one integration step, and assuming that the integration path begins from making all the transactions zero and raise them at the same rate to their actual value. Under these assumptions, the allocation of flow and losses to each transaction is given by (10).

$\bar{F}_{r t}=\phi_{r t}\left(T_{i j}\right) T_{i j}$

$\bar{L}_{r t}=\lambda_{r t}\left(T_{i j}\right) T_{i j}$

However, it is well known that

$L_{r} \approx \frac{1}{2} \sum_{t=1}^{N_{\mathrm{T}}} \bar{L}_{r t}$

where $N_{\mathrm{T}}$ is the number of transactions. The reconciliation process finds the new coefficients $L_{r t}^{\mathrm{c}}$ as shown in (11).

$L_{r t}^{\mathrm{c}}=\frac{L}{\sum_{r=1}^{N_{\mathrm{R}}} \sum_{t=1}^{N_{\mathrm{T}} \bar{L}_{r t}}} \bar{L}_{r t}$

where $N_{\mathrm{R}}$ is the number of branches of the grid and $L$ is the total losses of the system.
The coefficients $\bar{F}_{r t}$ do not lead, neither, to the correct flow of each line,

$F_{r} \neq \sum_{t=1}^{N_{\mathrm{T}}} \bar{F}_{r t}$

and a reconciliation process is also necessary, finding the corrected flow allocations by (12).

$F_{r t}^{\mathrm{c}}=\frac{F_{r}}{\sum_{t=1}^{N_{\mathrm{T}}} \bar{F}_{r t}} \bar{F}_{r t}$

It must be recalled that the reconciliation is made for adjusting the contributions of each transaction to the power flow of each branch. In the case of flows, these correction coefficients are close to one (except in low charged lines), due to the quasi-linear nature of the active power flow through lines.

This reconciliation process leads to very similar results than the integration in all the tested cases. An example will be shown in Section 5.1. This conclusion coincides with the analysis performed in [5] for the loss allocation problem. Therefore, the reconciliation process is recommended since it leads to similar accuracy and involves less computation time.

\section{Cost allocation}

Once the flow and losses have been allocated to the different transactions, it still remains the problem of allocating the cost to them. Throughout this section, it will be assumed that the cost recovery principle is applied, and therefore, the costs are allocated to each transaction according to the use that it makes of the grid, or to the losses that it produces. It must be also remarked that the flow and losses allocation may be positive or negative. If they are positive, it means that they contribute either to the dominant flow of the line, or to the losses.

The cost of losses may be allocated maintaining or not this positive or negative value. If the negative terms are allowed, a negative value of losses allocation would imply that this transaction should be paid for the losses that it avoids. The other possibility is not to allow negative contributions, by a translation of the slack node, as in [13], or by an allocation proportional to the absolute value, as in [14]. In this paper, the cost of losses are allocated allowing negative contributions, because it has been considered that it incentivizes those transactions that, in a given situation, reduce the overall system losses. Therefore, the final loss allocation to each transaction is given by Eq. (13).

$L_{t}=\sum_{r=1}^{N_{\mathrm{R}}} L_{r t}$

The cost of each branch may also be allocated in several ways to the branch users. If the negative terms are allowed, those flows opposed to the dominant flow should be paid. This could be an incentive for those transactions that decrease the branch flow, and could be adequate for heavily loaded systems. However, in lines with low flows due to heavy counterflows the method could lead to absurd results, because some transactions would receive a part or even all the price of the branch, and the rest of them 
should have to pay more than the cost of the branch in order to recover its cost. In this paper, it has been decided to allocate costs as shown in Eq. (14).

$U_{r t}=C_{r} \frac{\left|F_{r t}^{\mathrm{c}}\right|}{\sum_{t=1}^{N_{\mathrm{T}}}\left|F_{r t}^{\mathrm{c}}\right|}$

where $U_{r t}$ is the cost allocation of branch $r$ to the transaction $t$ and $C_{r}$ is the cost of branch $r$. With this allocation, there are no incentives to the counterflows, and the payments for the use of one branch is proportional to the use that the transaction makes of it in a given situation.

\section{Transactions}

When the market is based on bilateral contracts, the transactions are clearly defined. However, in pool systems, it is necessary to define them. This definition of transactions is a key issue because if affects decisively the results. Two principles are examined here, the Equivalent Bilateral Exchange principle and the Proportional Sharing Principle. The first one assumes that all the loads are supplied by all the generators proportionally to the power provided by them. This principle has been proposed in [4] and some aspects have been studied in [15]. The second one assumes that the outgoing power of each branch from a node comes from the incoming flows proportionally to the amount of these incoming flows. This principle has been proposed in [3], where it is also used for flow and losses allocation. In the proposed method the PSP principle will be only used for the definition of the transactions.

The EBE principle has the property of smaller variability with space and time [4]. Besides, as all the generation is supposed to supply to all the loads, the peripheral generation and load make more use of the grid than those located in the center of the system. They are, consequently, more charged. Another characteristic of this principle is that if it were applied to very large systems, it would lead to unrealistic results, since the relation between very distant nodes would ignore grid congestions or other constraints and non-linearities.

The Proportional Sharing Principle has advantages and disadvantages complementary to the EBE principle. First, it has more variability with time and space. Although this may be a disadvantage, it must be taken into account that in some cases the charges for grid use are computed throughout a long period - for instance, a year - and charged annually. Under these conditions, the volatility with time is less important.

Under this principle, the generation and demand located in the center of the system would likely supply or receive power to or from more nodes, and they would have greater relative charges than if EBE principle is followed. On the other hand, peripheral generation and demand are more confined and their charges tend to be smaller than under the EBE principle.

If this principle is applied to very large systems, it would split them into smaller balanced ones, so there are less interference with system non-linearities and congestions.

\section{Application examples}

This method has been applied to the IEEE-RTS96 system [16]. Two studies are shown here. The first one shows that the integration and the reconciliation processes lead to very similar results. The second one illustrates some already mentioned properties of the PSP and EBE principles for defining transactions in pool systems. Load flows have been run using the Matlab Power System Toolbox [17]. The IEEE-RTS96 1-area system is shown in Fig. 1. The sensitivities have been found adopting the solution (2) of Section 2.2, but it has been checked that the difference obtained with solution (3) is negligibly small.

\subsection{Integration versus reconciliation}

In this section, a comparison is shown between the results of the integration process with several steps, and those obtained in one integration step, with a subsequent reconciliation process. Both processes have been described in Section 2.3. The EBE principle has been used for transaction definitions. The integration path has been to assume all the transactions zero in the beginning, and to raise their level at the same rate up to their actual values.

As a measurement of accuracy of the integration process we use the reconciliation factors $\mathrm{RF}_{1}$ for losses and $\mathrm{RF}_{\mathrm{f}}$ for flows, as defined in Eq. (15)

$$
\begin{aligned}
\mathrm{RF}_{1} & =\frac{L}{\sum_{r=1}^{N_{\mathrm{R}}} \sum_{t=1}^{N_{\mathrm{T}}} \bar{L}_{r t}} \\
\mathrm{RF}_{\mathrm{f}} & =\frac{F}{\sum_{r=1}^{N_{\mathrm{R}}} \sum_{t=1}^{N_{\mathrm{T}}}\left|\bar{F}_{r t}\right|}
\end{aligned}
$$

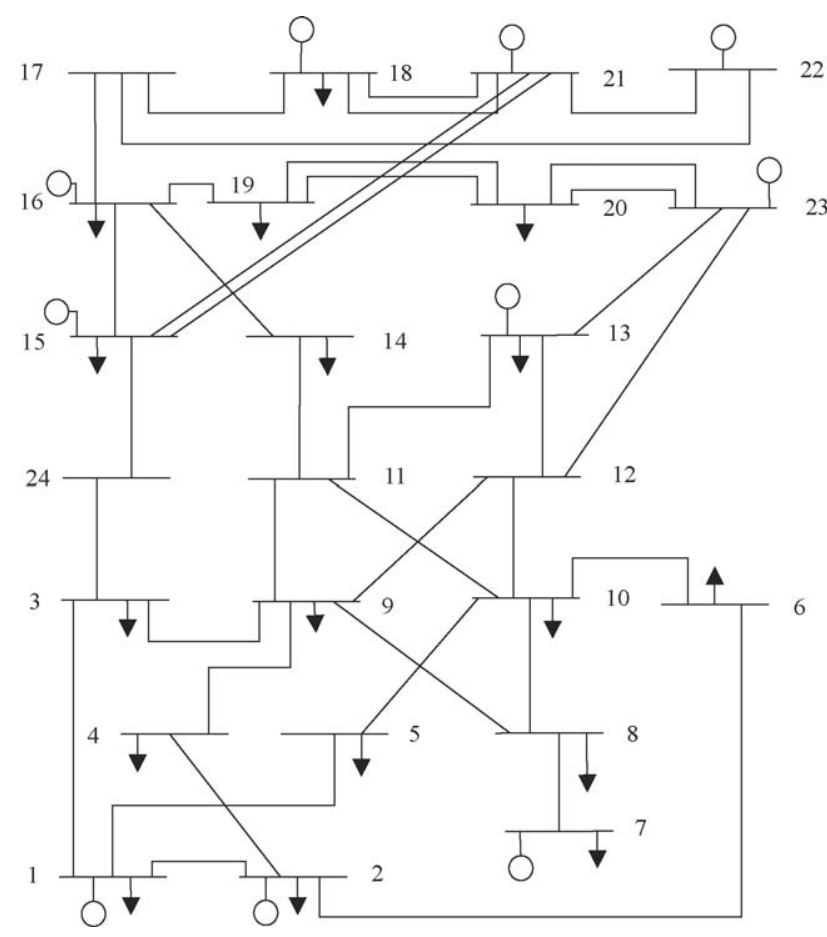

Fig. 1. IEEE-RTS96 system. 
Table 1

Reconciliation factors

\begin{tabular}{rll}
\hline Steps & $\mathrm{RF}_{\mathrm{l}}$ & $\mathrm{RF}_{\mathrm{f}}$ \\
\hline 1 & 0.4838 & 0.9563 \\
5 & 0.9878 & 0.9818 \\
10 & 1.0153 & 0.9825 \\
20 & 1.0211 & 0.9827 \\
50 & 1.0241 & 0.9828 \\
100 & 1.0243 & 0.9828 \\
\hline
\end{tabular}

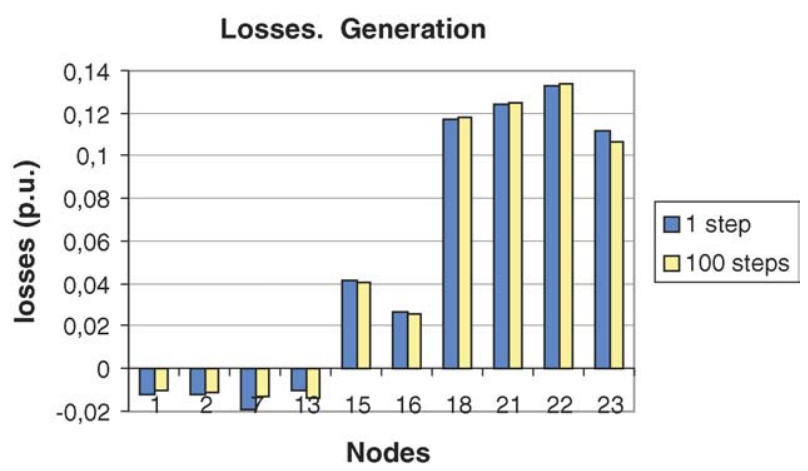

Fig. 2. Charge for losses among generation.

It is expected that the highest accuracy would lead to unity values, and that it would be obtained with a high number of integration steps. In Table 1, these factors are shown for different number of integration steps. The integration has been performed using the trapezoidal rule.

It is also interesting to compare the allocation between the single step case and the 100-steps case. This is shown in Fig. 2 for the losses allocated to the generators and in Fig. 3 for the losses allocated to the loads. Generation and demand are charged with half of the losses. Similar results are obtained for grid use.

From Table 1, it can be concluded that with few steps a reasonable accuracy is obtained, and that the increase in the number of steps does not lead to better behaviour. It must be remarked that the reconciliation factor for flows is similar to one in every case, underlining the quasi-linear nature of active power flowing through lines. Allocation is also very similar in both cases, as seen in Figs. 2 and 3, and negligible in comparison to the effect of different transaction definitions, as will be shown later. This reinforces the idea of using reconciliation instead of the inte-

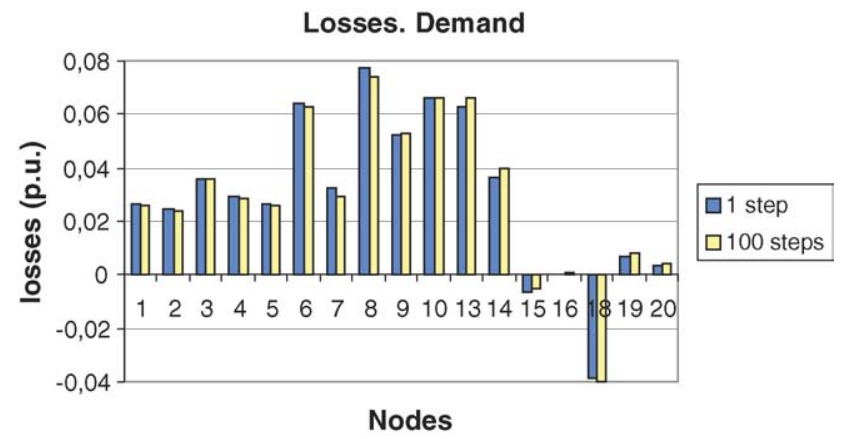

Fig. 3. Charge for losses among demand.

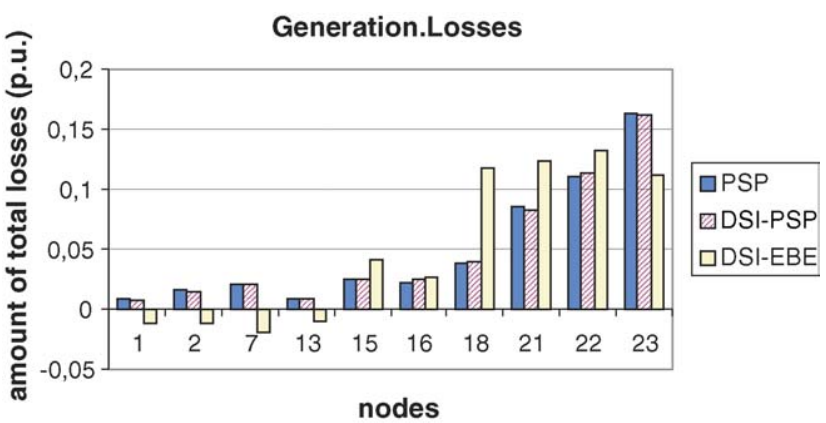

Fig. 4. Sharing of losses among generators (per power unit).

gration, since the computational burden is much smaller in the former case.

\subsection{Transaction definition effect}

In this section, the effect of choosing different transaction definitions is studied. In particular, the results of applying the EBE principle to transactions (method DSI-EBE) are compared to those obtained when transactions are defined using PSP (method DSI-PSP). Some of the previously mentioned properties of these principles are verified.

Both results have been compared with the application of the bare PSP method. It must be recalled that the difference between DSI-PSP and PSP methods is that, in the former, there exist sensitivities of every branch flow and losses to any transaction present in the systems, while in the latter there are lines not affected at all by many transactions. In all the cases, the costs have been equally divided between generation and demand.

In Fig. 4, the fraction of losses allocated to each generation node per unit MWh is shown. Fig. 5 shows the same concept for the demand. Figs. 6 and 7 show the use of grid assigned to each generation and demand node, respectively, per unit MWh. The following conclusions could be extracted from these results:

- In general, it can be observed that the charge for the use of grid is more uniform using the EBE principle than using the PSP principle. This is because the use of every element of the grid is shared among more users with EBE transactions than with the PSP.

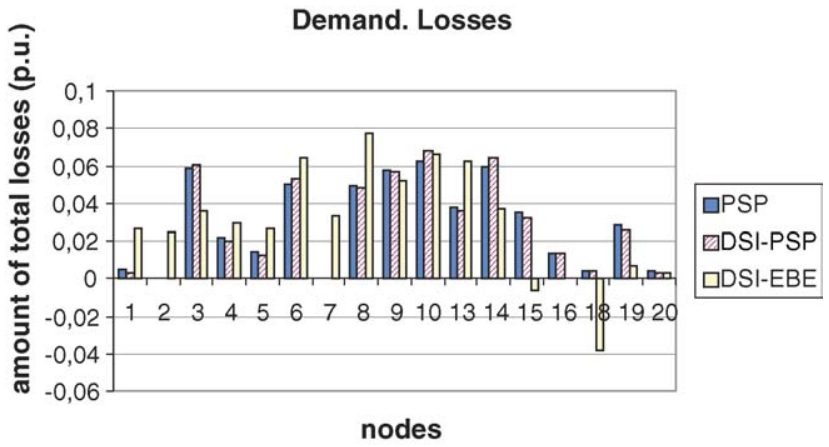

Fig. 5. Sharing of losses among demand (per power unit). 


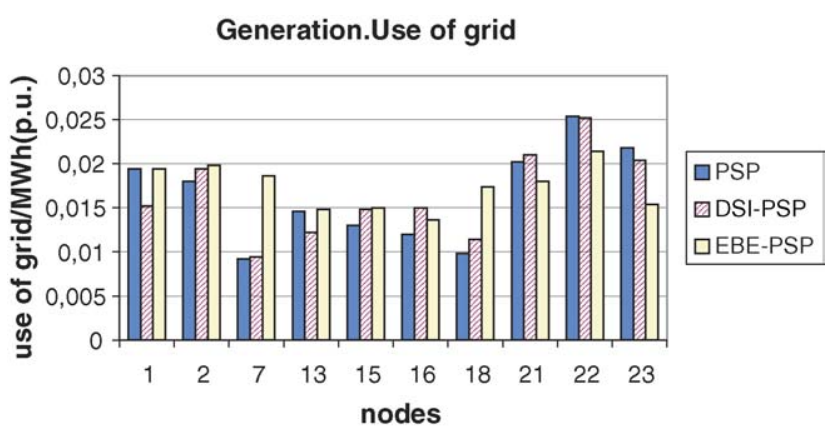

Fig. 6. Charge for use of grid among generators (per power unit).

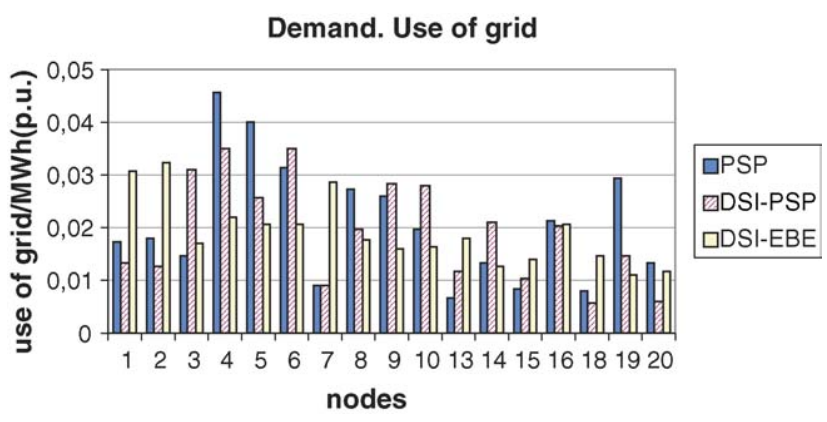

Fig. 7. Charge for use of grid among demand (per power unit).

- With regard to losses, it could be observed that, since most of the loads are located in the "southern" region of the system while the generation is mostly located in the "north", the dominant flow of power is from "north" to "south", and the flows opposed to it are allocated negative losses. Hence, under EBE assumption, "southern" generation nodes such as 1, 2, 7 and 13 are allocated negatives losses while the demand of nodes $15,16,18-20$ are allocated very low or negative terms. However, under PSP, these transactions do not exist, or have a small value, and these nodes are allocated positive amounts, since the couples generation-demand are closer between them.

- Peripheral generators 1,7 and 18 are more charged under EBE principle than if PSP is assumed. The same happens with demand in nodes 1,2 and 7. On the other hand, central nodes, as 4-6, 9 and 10 in demand are more charged under PSP. Generation in nodes 21-23 are the big suppliers in both cases,
Table 2

EBE transactions

\begin{tabular}{|c|c|c|c|c|c|c|}
\hline \multirow[t]{2}{*}{ Area } & \multicolumn{2}{|l|}{ Area 1} & \multicolumn{2}{|l|}{ Area 2} & \multicolumn{2}{|l|}{ Area 3} \\
\hline & Losses & Grid use & Losses & Grid use & Losses & Grid use \\
\hline 1 & 0.6426 & 0.5291 & 0.1906 & 0.2357 & 0.1642 & 0.2018 \\
\hline 2 & 0.1919 & 0.2437 & 0.6457 & 0.5513 & 0.1641 & 0.1982 \\
\hline 3 & 0.1592 & 0.2273 & 0.1693 & 0.2132 & 0.671 & 0.5996 \\
\hline
\end{tabular}

Payments from areas in rows to the areas in columns.

but in the case of PSP transactions they are more charged because they must supply relatively more power to distant loads. The reduction for generator 18 with PSP is because of the demand in the same node that is mostly supplied by the generation at that node under PSP assumptions.

- While there are small differences between loss allocation with PSP and with DSI-PSP methods, there are substantial differences between them in grid use allocation. These differences are more apparent for the demand than for the generation. This is because there are less generators than loads, and therefore the latter have less "paths" linking them to generators. PSP method (unlike DSI-PSP) does not consider the influence that a transaction may have on lines that are not in the path that links the generation and demand in such transaction.

As a final example, let us consider the complete (three zones) IEEE-RTS96 system. In this example, the three systems are almost exactly the same, and they are all balanced in generation and load. Only exchange loop flows take place between them. These exchanges (active power in p.u.) may be seen in Fig. 8, where the number of the nodes in the systems has been preceded by another number indicating its area belong.

In this situation, if the EBE principle is used for defining the transactions, the part of the cost of each grid and losses that would have to pay the users of each zone (rows) to the other zones (columns) is given in Table 2. Table 3 shows the same concept, but when the PSP is used for defining the transactions. It can be observed that PSP splits the whole grid into its balanced subsets, while the EBE principle divides the overall cost among all users. It is dubious whether the allocation following EBE principles is adequate for large systems, like real power networks.

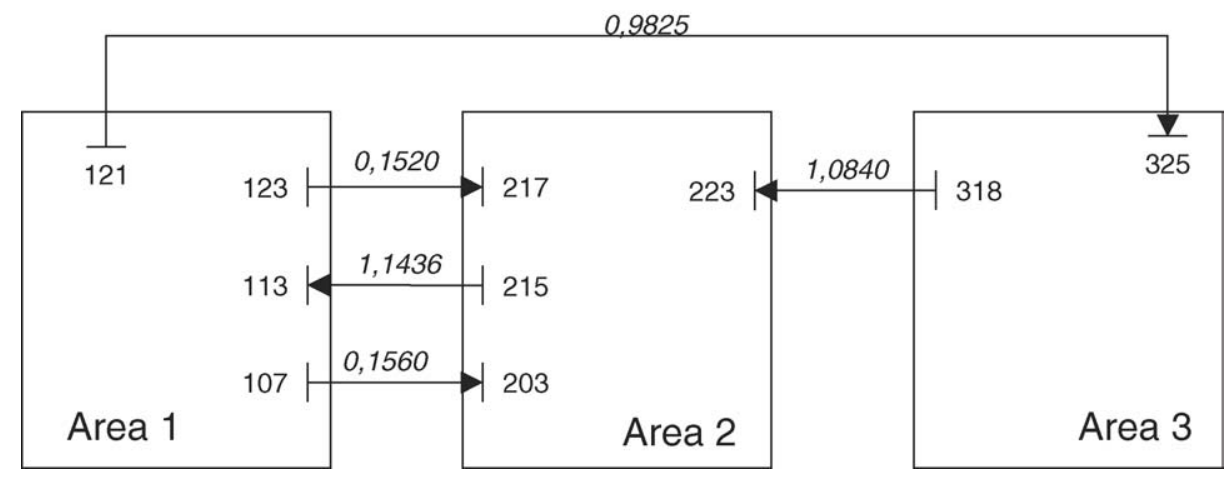

Fig. 8. Active power exchanges in the IEEE-RTS96 3-area system. Active power flows in p.u. 
Table 3

PSP transactions

\begin{tabular}{|c|c|c|c|c|c|c|}
\hline \multirow[t]{2}{*}{ Area } & \multicolumn{2}{|l|}{ Area 1} & \multicolumn{2}{|l|}{ Area 2} & \multicolumn{2}{|l|}{ Area 3} \\
\hline & Losses & Grid use & Losses & Grid use & Losses & Grid use \\
\hline 1 & 0.8989 & 0.8222 & 0.0631 & 0.0982 & 0.023 & 0.0542 \\
\hline 2 & 0.0826 & 0.1242 & 0.9134 & 0.8481 & 0.0078 & 0.0334 \\
\hline 3 & 0.0118 & 0.0539 & 0.0267 & 0.0532 & 0.9708 & 0.9126 \\
\hline
\end{tabular}

Payments from areas in rows to the areas in columns.

\section{Conclusion}

From the presented results, it can be concluded that:

- The problems of losses and flow allocation to transactions, cost allocation and transaction definition can be solved independently.

- Differential methods seem adequate to solve the flow and losses allocation problems, because they take into account the physical laws of electric grids. A method for solving this problem has been proposed. This method does not depend on the choice of the slack bus and uses the AC load flow equations.

- Although the integration of the differential equation seems to be theoretically sound, it needs unrealistic assumptions or, at least, to define clearly initial conditions and an integration path. Besides, a reconciliation process involving much less computational burden provides the same results.

- Cost allocation methods have been also discussed. It has been proposed to allow negative allocations for losses, and to not allow them in flow allocation, since it could lead to unacceptable results.

- Transaction definition has a key role in the overall allocation process. EBE principle charges more the peripheral generation and demand, and seems to be a bad choice for large systems. PSP is more volatile spatially and temporally, charges more the central generation and demand and tends to split a big system into several balanced subsystems.

\section{Acknowledgments}

Thanks are due to Red Electrica de España for their support to this work. Jose Luis Fernandez Gonzalez is also gratefully acknowledged for useful comments.

\section{Appendix A. Angle and voltage sensitivities to transactions with different slack buses}

In Fig. 9, a schematic electric system is shown. The differential transaction $\mathrm{d} T_{i j}$ consists in an active power $\mathrm{d} P_{i j}$ injected in node $j$ and delivered in node $i$.

When the slack bus is node o, the additional losses produced by this transaction $\mathrm{d} P_{1}$ are provided by this node $\mathrm{o}$. The sensitivity of voltage angle of node $n$ to this differential increase of

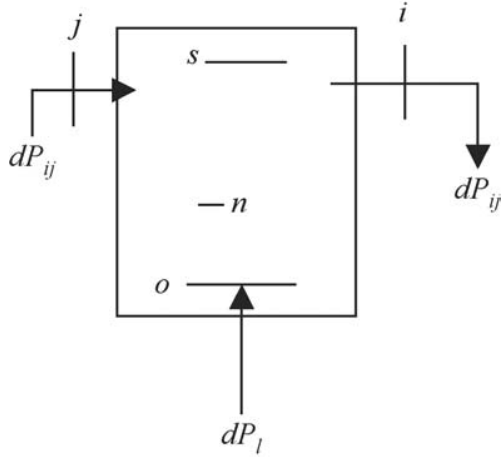

Fig. 9. Electric system.

the transaction, $\left.\frac{\partial \delta_{n}}{\partial T_{i j}}\right|_{\mathrm{o}}$ is given by (16):

$\left.\frac{\partial \delta_{n}}{\partial T_{i j}}\right|_{\mathrm{o}}=\left.\frac{\partial \delta_{n}}{\partial P_{j}}\right|_{\mathrm{o}}-\left.\frac{\partial \delta_{n}}{\partial P_{i}}\right|_{\mathrm{o}}$

If, however, the slack bus is the node s, the variation of angle $\mathrm{d} \delta_{n}$ when the same transaction $\mathrm{d} T_{i j}$ takes place, will have the same value only if the losses produced by this transaction are also supplied by the bus o. In this case, this sensitivity could be written as (17)

$\left.\frac{\partial \delta_{n}}{\partial T_{i j}}\right|_{\mathrm{s}}=\left.\frac{\partial \delta_{n}}{\partial P_{j}}\right|_{\mathrm{s}}-\left.\frac{\partial \delta_{n}}{\partial P_{i}}\right|_{\mathrm{s}}+\left.\frac{\partial \delta_{n}}{\partial P_{\mathrm{s}}}\right|_{\mathrm{s}} \frac{\mathrm{d} P_{1}}{\mathrm{~d} P_{i j}}$

As (16) and (17) give the same results, Eq. (9) is demonstrated.

In order to make the method fully slack invariant, two solutions have been proposed in Section 2.2. Solution (2) makes node $j$ the slack bus for calculating the sensitivities to this transaction $\mathrm{d} T_{i j}$. This means that the incremental losses $\mathrm{d} P_{1}$ are supplied by generator $j$. As this is performed for each transaction, the sensitivity coefficients do not depend on the choice of the initial slack bus (o or s in this case). However, the power injected in node $j$ is not the same as that taken in node $i$. Solution (3) makes all the generators but $j$ to provide the losses $\mathrm{d} P_{1}$. It is also fully slack invariant, and in this case the power taken in $i$ is the same that the injected in $j$.

\section{Appendix B}

\section{List of symbols}

$b_{k m} \quad$ susceptance of line between nodes $k$ and $m$

$C_{r} \quad$ cost of branch $r$

$F_{r} \quad$ average active power flow in branch $r$

$F_{r t} \quad$ average active power flow in branch $r$ allocated to transaction $t$

$\bar{F}_{r t} \quad$ average active power flow in branch $r$ allocated to transaction $t$, before the reconciliation process

$F_{r t}^{\mathrm{c}} \quad$ average active power flow in branch $r$ allocated to transaction $t$, after the reconciliation process

$g_{k m} \quad$ conductance of line between nodes $k$ and $m$

$I_{\mathrm{P}} \quad$ integration path

$L \quad$ system losses

$L_{r} \quad$ losses in branch $r$ 
$L_{r t} \quad$ losses in branch $r$ allocated to transaction $t$

$\bar{L}_{r t} \quad$ losses in branch $r$ allocated to transaction $t$, before the reconciliation process

$L_{r t}^{\mathrm{c}} \quad$ losses in branch $r$ allocated to transaction $t$, after the reconciliation process

$N_{\mathrm{R}} \quad$ number of branches

$N_{\mathrm{T}} \quad$ number of transactions

$P_{i} \quad$ power injected in node $i$

$P_{i j} \quad$ power exchanged in transaction $T_{i j}$

$T_{i j} \quad$ transaction between the generator $j$ and the load $i$

$u_{k} \quad$ voltage in node $k$

$U_{r t} \quad$ cost of branch $r$ allocated to transaction $t$

$\delta_{k} \quad$ voltage angle in node $k$

$\phi_{r t} \quad$ sensitivity of average active power in branch $r$ to a variation of transaction $t$

$\lambda_{r t} \quad$ sensitivity of losses in branch $r$ to a variation of transaction $t$

\section{References}

[1] J.W. Marangon Lima, Allocation of transmission fixed charges: an overview, IEEE Trans. Power Syst. 11 (August (3)) (1996) 14091418.

[2] J.I. Pérez Arriaga, F.J. Rubio, J.F. Puerta, Marginal pricing of transmission services: an analysis of cost recovery, IEEE Trans. Power Syst. 10 (February (1)) (1995) 546-553.

[3] J. Bialek, Tracing the flow of electricity, IEE Proc.-Gener. Transm. Distrib. 143 (July (4)) (1996) 313-320.

[4] F.D. Galiana, A.J. Conejo, H. Gil, Transmission network cost allocation based on equivalent bilateral exchanges, IEEE Trans. Power Syst. 18 (November (4)) (2003) 1425-1431.
[5] F.D. Galiana, A.J. Conejo, I. Kockar, Incremental transmission loss allocation under pool dispatch, IEEE Trans. Power Syst. 17 (February (1)) (2002) 26-33.

[6] F.D. Galiana, M. Phelan, Allocation of transmission losses to bilateral contracts in a competitive environment, IEEE Trans. Power Syst. 15 (February (1)) (2000) 143-150.

[7] A.M. Leite da Silva, J.G. de Carvalho Costa, Transmission loss allocation: Part I-single energy market, IEEE Trans. Power Syst. 18 (November (4)) (2003) 1389-1394.

[8] J. Mutale, G. Strbac, S. Curcic, N. Jenkins, Allocation of losses in distribution systems with embedded generation, IEE Proc.-Gener. Transm. Distrib. 147 (January (1)) (2000) 7-14.

[9] A.J. Conejo, F.D. Galiana, I. Kockar, Z-Buss loss allocation, IEEE Trans. Power Syst. 16 (February (1)) (2001) 105-110.

[10] Z.Q. Wu, Determination of the branch power and loss caused by node injection power, IEE Proc.-Gener. Transm. Distrib. 150 (November (6)) (2003) 722-726.

[11] A.J. Conejo, N. Alguacil, G. Fernández Ruiz, Allocation of the cost transmission losses using a radial equivalent network, IEEE Trans. Power Syst. 18 (November (4)) (2003) 1353-1358.

[12] A.J. Wood, B.F. Wollenberg, Power Generation Operation and Control, John Wiley \& Sons, New York, 1996.

[13] A.J. Conejo, J.M. Arroyo, N. Alguacil, A.L. Guijarro, Transmission loss allocation: a comparison of different practical algorithms, IEEE Trans. Power Syst. 17 (August (3)) (2002) 571-576.

[14] G. Gross, S. Tao, A physical-flow-based approach to allocating transmission losses in a transaction framework, IEEE Trans. Power Syst. 15 (May (2)) (2000) 631-637.

[15] C. Vázquez, L. Olmos, J.I. Pérez Arriaga, On the selection of the slack bus in mechanisms for transmission network cost allocation that are based on network utilization, in: Proceedings of the 15th Power Systems Computing Conference, PSCC, Seville, 2002.

[16] The IEEE reliability test system, IEEE Trans. Power Syst. 14 (August (3)) (1999) 1010-1018

[17] Power System Toolbox, Version 2.0, Cherry Tree Scientific Software. 